\title{
Médiévales
}

Langues, Textes, Histoire

78 | printemps 2020

Moyen Âge en séries

\section{Thierry la Fronde, figure de la Résistance}

Quand une Occupation en cache une autre...

\section{Bernard Papin}

\section{(2) OpenEdition}

\section{Journals}

Édition électronique

URL : https://journals.openedition.org/medievales/10787

DOI : $10.4000 /$ medievales. 10787

ISSN : 1777-5892

Éditeur

Presses universitaires de Vincennes

\section{Édition imprimée}

Date de publication : 20 août 2020

Pagination : 69-86

ISBN : 978-2-37924-093-5

ISSN : 0751-2708

\section{Référence électronique}

Bernard Papin, «Thierry la Fronde, figure de la Résistance », Médiévales [En ligne], 78 | printemps 2020,

mis en ligne le 02 janvier 2022, consulté le 22 avril 2022. URL : http://journals.openedition.org/

medievales/10787; DOI : https://doi.org/10.4000/medievales.10787 
Bernard Papin

\section{Thierry la Fronde, figure de la Résistance \\ Quand une Occupation en cache une autre...}

Dans les années 1950 et 1960 du XXe siècle, au sortir de la guerre et de la Résistance, les responsables de la RTF puis de l'ORTF ${ }^{1}$ inventent

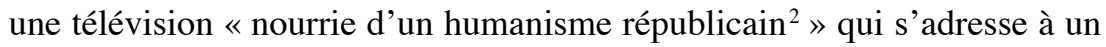
public «pensé comme citoyen ${ }^{3}$ » qu'il faut instruire tout en le divertissant par le truchement d'adaptations des grandes œuvres du patrimoine littéraire français et mondial et de grandes dramatiques historiques ${ }^{4}$. Sous l'impulsion de directeurs de programmes ${ }^{5}$ animés d'une telle ambition, les réalisateurs de la jeune télévision française mettent leur «art de la télévision ${ }^{6}$ » au service d'une «utopie culturaliste ${ }^{7}$. La fiction historique est évidemment un vecteur privilégié de cette volonté de transmission et d'éducation, voire d'édification. En 1963, une série médiévale destinée initialement au jeune public connaît un très vif succès : Thierry la Fronde (1963-1966) conte les aventures d'un jeune noble, Thierry de Janville, qui s'oppose - nous sommes au XIV ${ }^{\mathrm{e}}$ siècle - à l'occupation anglaise de la Sologne pendant la guerre de Cent Ans. Jean-Claude Drouot incarne le personnage pendant cinquante-

1. L'Office de radiodiffusion-télévision française (1964-1974) succède en 1964 à la Radiodiffusion-télévision française (1949-1964). Sur la création de l'«Office» et sur ce qu'implique ce changement de statut, voir M. SAUVAGE et I. VEYRAT-MASSON, Histoire de la télévision française : de 1935 à nos jours, Paris, 2014, p. 81-82.

2. M.-F. LÉVY, «Télévision, publics, citoyenneté. 1950-1974 », dans É. COHEN et M.F. LÉvy éd., La Télévision des Trente Glorieuses. Culture et politique, Paris, 2007, p. 91-111 (p. 97).

3. Ibid., p. 92.

4. Avec, notamment, La caméra explore le temps, 1957-1966.

5. Jean d'Arcy de 1952 à 1959, Albert Ollivier de 1959 à 1964.

6. Cf. G. Delavaud, L'Art de la télévision. Histoire et esthétique de la dramatique télévisée (1950-1965), Bruxelles, 2005.

7. B. PAPIN, «L'utopie culturaliste de la Télévision nationale populaire », dans J.M. ClerC éd., Cinéma, littérature, adaptations, Montpellier, 2005, p. 233-253. 
deux épisodes et devient très rapidement une des figures majeures de cette télévision qui promeut alors des héros «fougueux, braves et généreux ${ }^{8}$ ». Cette série - légèrement feuilletonnante au sens où elle épouse, en dépit de la fermeture de chaque épisode sur lui-même, une certaine chronologie historique, nous y reviendrons - est une fiction historique pour enfants et adolescents qui reprend la veine médiévale qu'avait inaugurée, avec succès, en 1959, la série britannique Ivanhoé.

Il s'agit assurément, en ce début des années 1960, de promouvoir auprès du jeune téléspectateur des héros français - comme Bayard ou Thibaud ultérieurement ${ }^{9}$ - mais également de concurrencer le succès des premières séries venus d'Outre-Atlantique qui proposent un modèle westernien jugé exotique et souvent trop violent ${ }^{10}$. Et pour les « hussards noirs ${ }^{11} »$ du service public de télévision, l'aspect éducatif des fictions jeunesse ne peut être négligé. Thierry la Fronde sera donc une fiction (relativement) documentée et devra donner l'exemple d'un héros positif, qui pratique une violence de rétorsion toujours proportionnée.

Très vite cependant, ce Robin des Bois solognot fédère autour de l'écran alors très familial un public beaucoup plus large que celui initialement visé. Et cette production aux moyens limités ${ }^{12}$, de facture plutôt inégale en termes de scénario et de mise en scène, devient un immense succès - d'où la déception, voire la colère, du public après le renoncement de JeanClaude Drouot à l'issue de la quatrième «saison » (comme on ne disait pas encore) qui met fin du même coup à une série qu'il incarnait trop pour qu'elle perdurât après son départ ${ }^{13}$ - avant de s'inscrire dans la mémoire des enfants du baby-boom et d'entrer «de plain-pied dans la légende télévisuelle ${ }^{14} »$. Comment expliquer que cette série, que ses promoteurs trouvent eux-mêmes parfois médiocre et qui semble aujourd'hui si éloignée des standards de qualité de nos séries favorites actuelles ${ }^{15}$, ait pu connaître

8. Télérama, $\mathrm{n}^{\circ} 720,3$ novembre 1963 , présentant Thierry la Fronde.

9. Le Chevalier Bayard, 1964 ; Thibaud ou Les Croisades, 1968-1969.

10. Par exemple, Roy Rogers (1962) ne connaîtra pas de seconde saison après les courriers de protestation véhéments adressés à la RTF. Voir Pierrefitte-sur-Seine, AN, Fonds ORTF, 20130366/26.

11. J. BOURDON, «Les racines du mythe», Quaderni, 65 (2007-2008), dossier «L'ambivalence du mythe de l'ORTF », p. 9-19 (p. 12).

12. Du moins au début car, du fait du succès de la première série de treize épisodes, les chevaux font leur apparition dans la deuxième. Un luxe qu'autorise désormais l'augmentation de budget...

13. En vertu de «l'étrange nature du héros de série télévisée »: voir S. CHALvonDEMERSAY, «Enquête sur l'étrange nature du héros de série télévisée », Réseaux, 165 (2011), p. 181-214.

14. J. BAUdOU et J.-J. SCHLERET, Les Feuilletons historiques de la télévision française, de Thierry la Fronde à Maria Vandamme, Paris, 1992, p. 26.

15. Cf. F. Jost éd., Pour une télévision de qualité, Bry-sur-Marne, 2014. 
un tel succès ? Comment cette fantaisie historique a-t-elle pu susciter un tel engouement?

Nous voudrions ici montrer que cette production française, qui transporte le modèle littéraire du hors-la-loi justicier de la forêt de Sherwood à celle de Sologne, évoque aussi une autre Occupation. Quinze ans après la fin de la Seconde Guerre mondiale, la France baigne encore dans un « résistancialisme ${ }^{16}$ » de bon (ou de mauvais) aloi, et la résistance de Thierry évoque une histoire nettement plus proche et plus sensible pour le public de la jeune télévision. Derrière le mythe littéraire et cinématographique du gentil hors-la-loi défenseur des opprimés et désireux de «bouter » les Anglais hors de France, se profile une autre historicité, alors bien plus contemporaine.

\section{Une légende télévisuelle}

Thierry la Fronde fait partie de ces co-productions que la RTF multiplie au tournant des années 1960 pour augmenter et diversifier son offre de programmes. Cette collaboration avec la société de production Télé-France-Films (aujourd'hui Telfrance) a déjà permis à la télévision française de connaître ses premiers grands succès dans la catégorie de la fiction sérielle avec, entre autres, Le Temps des copains ${ }^{17}$ et Janique Aimée ${ }^{18}$, deux feuilletons diffusés avant le journal télévisé et qui connurent l'un et l'autre un succès phénoménal. Une convention est signée entre les deux parties en avril 1963 pour une première série de douze épisodes, pour un coût de 80000 francs par épisode ${ }^{19}$. La RTF est partie prenante aux deux tiers dans le financement et dispose du droit de «faire apporter aux scénarios, à la mise en scène et à la distribution artistique, toutes les modifications qu' [elle] jugera utiles ${ }^{20} \gg$.

Sur le marché de la production télévisuelle française, Thierry la Fronde a d'abord été conçu pour contrebalancer les séries étrangères,

16. Cf. H. Rousso, Le Syndrome de Vichy. De 1944 à nos jours, Paris, 1990.

17. Diffusion en 1962-1963. Réalisé par Robert Guez, qui réalise également les premiers épisodes de Thierry la Fronde.

18. Diffusion en 1963. Scénario de Jacques Siclier.

19. Environ un peu plus de 110000 euros aujourd'hui [http://leparticulier.lefigaro.fr/ jcms/p1_1553305/le-tableau-de-conversion-en-francs/euros-est-actualise], ce qui est peu par rapport aux normes en vigueur actuellement pour une production en costumes. À titre de comparaison, chaque épisode de la série policière historique Nicolas Le Floch, diffusée sur France 2 à compter de 2008, coûtait environ deux millions d'euros.

20. Article 2. Dossier de production: TLF, 1963, RTF, Convention de coproduction signée le 18/4/63, consultation à l'Inathèque sous dérogation. 
britanniques ${ }^{21}$ ou américaines ${ }^{22}$, qui occupent alors avec succès le créneau des émissions destinées à la jeunesse. Dans un contexte où les productions américaines sont parfois perçues comme l'avant-garde d'un impérialisme économique et culturel, la bataille fait rage en ce début des années 1960 entre «séries» américaines et «feuilletons français ${ }^{23}$ » et, comme de Janique Aimée, Télé 7 jours peut dire de Thierry la Fronde que c'est une fiction «bien de chez nous ${ }^{24} »$. Dans la grande lignée, littéraire d'abord, cinématographique ensuite, des redresseurs de torts au grand cœur, Thierry va soutenir l'honneur de la production nationale. Au panthéon des héros «sans peur et sans reproche » qui deviennent hors-la-loi pour mieux concourir à la restauration de la loi et de l'ordre juste, il faut qu'il impose son nom : son créateur, Jean-Claude Deret, souligne à la fois la concurrence héroïque à laquelle son personnage est confronté et la difficulté à lui trouver un attribut qui lui soit propre: «Qu'est-ce qu'ils m'avaient laissé, les autres ? Robin des Bois avait son arc, Lancelot, il avait son épée, Guillaume Tell, il avait son arbalète... mais personne n'avait pensé à la fronde ${ }^{25}$. » Une arme utilisée au Moyen Âge, précise Jean-Claude Deret, et qui est l'arme «du peuple alors que l'épée est l'arme des nobles ${ }^{26} »$. Comme ses modèles, «c'est un gentil, c'est un pur », qui «n'a pas de mauvaises pensées ${ }^{27}$ » et qui doit pouvoir être proposé en exemple au jeune public. À partir de 1965, Thierry la Fronde devra cependant affronter la concurrence d'un autre héros du même type, venu d'Amérique et fort populaire dans la même décennie : Zorro, un noble qui, comme lui, doit travestir son identité et s'avancer masqué pour mieux défendre les faibles.

$\mathrm{Si}$, dès 1964, « la fronde du jeune homme en collant» apparaît comme « une arme bien légère » par rapport à « la mitraillette de l'homme au chapeau $\mathrm{mou}^{28} »$, , l'incorruptible » Eliot Ness, autre concurrent sérieux

21. Comme Ivanhoé, autre série médiévale déjà citée, première diffusion française en 1959.

22. Par exemple, Rintintin, série diffusée en France à partir de 1958.

23. Voir à ce sujet, S. LE PAJOLEC, «Comment Steve McQueen devint une star de la télévision française. Le lancement d'Au nom de la loi », dans B. PAPIN, M. TSIKOUNAS et S. ChALVON-DEMERSAY éd., Fictions sérielles au temps de la RTF et de l'ORTF (19491974), Paris, 2018, p. 113-129.

24. Télé 7 jours, $\mathrm{n}^{\circ} 150,2$ février 1963, cité par S. LE PAJOLEC, «Comment Steve McQueen... », p. 117.

25. Dans un entretien accordé à Sabine Chalvon-Demersay dans le cadre de la réalisation de la collection Histoires de fiction, série documentaire télévisuelle de 6 × 52 minutes de S. Chalvon-Demersay et P. Jeudy, France 5, 2003-2004. Nous remercions ici Sabine ChalvonDemersay qui a bien voulu nous confier l'intégralité de cet entretien. Dans la suite de cet article, cet entretien sera ainsi référencé : « Entretien JCD/SCD ».

26. Télérama, $\mathrm{n}^{\circ} 720,3$ novembre 1963, p. 17.

27. « Entretien JCD/SCD».

28. I. VEYRAT-MASSON, «Une fronde contre Eliott Ness », Le Temps des médias, 13/2 (2009), p. 12. 
à la notoriété auprès du public ${ }^{29}$, la série connaît un succès immédiat et massif, comme de nombreuses œuvres de l' " âge d'or des feuilletons » qui fédèrent autour du poste de télévision et de son unique chaîne (jusqu'en 1964) de 60 à $70 \%$ des téléspectateurs potentiels, avec, régulièrement, des audiences de « 17 à 19 millions de téléspectateurs ${ }^{30} »$. Jean-Claude Drouot devient très vite une des premières vedettes de la télévision : après à peine deux épisodes, Télé 7 jours lui consacre sa couverture ${ }^{31}$ et introduit la confusion entre le personnage et son interprète («Thierry la Fronde papa »), une confusion qui témoigne de l'immédiate adhésion du public au personnage et à ce qu'il incarne ${ }^{32}$. Très vite également, un public plus adulte a rejoint celui des jeunes téléspectateurs ${ }^{33}$. Signe de cet engouement, les premiers «produits dérivés » de l'histoire de la télévision française font leur apparition, les clubs des amis de Thierry la Fronde se multiplient ${ }^{34}$ et le justicier solognot a droit, après une seule saison, à un «épisode tragico-comique », «Le collier de Janville », dans le cadre de «La revue des feuilletons » du «festival de Noël $1963 »$, une série de pastiches et/ ou de parodies où Thierry la Fronde se retrouve en belle compagnie, avec les grands succès feuilletonnants de l'époque, Le Temps des copains, Le Chevalier de Maison-Rouge $e^{35}$, Janique Aimée...

Dans un tel contexte, on comprendra aisément le désarroi de tous les fans de Jean-Claude Drouot quand celui-ci, après quatre séries de treize épisodes et toujours le même succès, annonce qu'il abandonne la série. En 1973, le jeune comédien justifiera sa décision par le caractère « encombrant » d'un personnage devenu «mythe » dont il lui importait de se séparer avant qu'il ne soit trop tard pour sa carrière de comédien, avant d'ajouter, pleinement conscient de la déception ainsi suscitée à l'époque :

29. Les Incorruptibles, série diffusée en France à partir de 1964. Cette série qui sera diffusée très vite en deuxième partie de soirée avec carré blanc était en revanche réputée réservée aux adultes.

30. G. PoELS, «L'audience des fictions sérielles dans les années 1960 et 1970 : la mesure de l'âge d'or », dans B. PAPIN, M. TsiKounas et S. ChaLVON-DEMERSAY éd., Fictions sérielles..., Paris, 2018, p. 39-48 (p. 41).

31. Télé 7 jours, $\mathrm{n}^{\circ} 191,16$ novembre 1963.

32. S. ChALVON-DEMERSAY, «L'homme qui voulait être acteur, Thierry la Fronde », Télévision, 6 (2015), p. 81-97 (p. 95).

33. Cet élargissement du public n'a rien d'inédit à cette époque de chaîne unique et de public familial (d'où certaines contraintes en termes de représentation, de la violence notamment). Un même phénomène s'observe à propos du Théâtre de la jeunesse (19601968) de Claude Santelli. Ce dernier revendiquait cette diversité des publics et la pluralité des lectures en fonction des âges.

34. G. POELS, «L'audience des fictions sérielles... », p. 43.

35. Diffusion en 1963, réalisation Claude Barma. 
«On ne comprenait pas pourquoi je refusais aux enfants, aux gens, de ne pas continuer. Et pour moi, c'était nécessaire ${ }^{36}$. »

En 1991, une autre parodie - celle des Inconnus avec « Thierryla-France ${ }^{37} \gg-$ témoignera, avec une tendre férocité, de la persistance du mythe télévisuel dans l'inconscient collectif des téléspectateurs français. De même, des remakes cinématographiques sont régulièrement annoncés ${ }^{38} \ldots$ Aucun, à ce jour, n'a abouti : preuve sans doute que le «héros de série télévisée » se distingue «par le lien particulièrement ferme établi entre un personnage et un corps ${ }^{39}$ » et qu'il n'est pas aisé d'incarner à nouveau à l'écran une figure à ce point ancrée dans la mémoire collective.

\section{Une fiction historique}

En dépit du caractère captif du public et du goût pour les feuilletons ${ }^{40}$, ce succès peut sembler aujourd'hui déconcertant et, un demi-siècle plus tard, les analystes ne sont guère tendres envers cette production jugée pour le moins inégale, «tournée à bride abattue, sans grand sens artistique, sur des scénarios simplistes et répétitifs ${ }^{41} \gg$. Mais à l'époque, déjà, les avis sont partagés : Jean-Claude Drouot n'a jamais caché ses réticences à l'égard de son personnage qu'il trouvait trop peu complexe ${ }^{42}$, et même les responsables de la RTF ou de l'ORTF n'ont pas toujours été très enthousiastes : en 1965, une «note de projection » avant la diffusion de la dernière série de treize épisodes parle d'une « série honorable en progrès », avec deux épisodes jugés «médiocres » et sept «moyens », comportant encore trop de dialogues au détriment des scènes d'action ${ }^{43} \ldots$

Comment expliquer qu'une fiction jugée assez unanimement médiocre ait pu entrer ainsi en résonance avec le public des années 1960 ? Au-delà des effets de fascination que provoquait la conquête progressive de l'espace domestique par la nouvelle «boîte à images », les Français ont-ils été séduits par un récit historique, comme la télévision de l'époque

36. [https://www.ina.fr/contenus-editoriaux/articles-editoriaux/jean-claude-drouot-1inoubliable-thierry-la-fronde/]

37. [https://www.youtube.com/watch?v=LYUzklRmSjI]

38. Le dernier en date, sans doute, est celui de Philippe Duguay en 2012. Comme d'autres avant lui, celui-ci n'a pas abouti, vraisemblablement pour des problèmes de droits. Dossier de production : TLF, 1963, RTF, consultation à l'Inathèque sous dérogation.

39. S. CHALVON-DEMERSAY, « L'homme qui voulait être acteur... », p. 95.

40. Néanmoins, à côté des grands succès que nous avons cités et de bien d'autres, il y eut aussi en cet « âge d'or » des échecs retentissants.

41. Article «Thierry la Fronde», dans N. C. AHL et B. FAU éd., Dictionnaire des séries télévisées, Paris, 2016.

42. Voir ses propos très critiques rapportés dans J. BAUDOU et J.-J. SCHLERET, Les Feuilletons historiques..., p. 25-26.

43. Pierrefitte-sur-Seine, AN, Fonds ORTF, 20130366/199. 
savait régulièrement, et avec succès, leur en proposer ? Les caméras explorent alors sans relâche le passé national, mobilisent les consciences citoyennes ${ }^{44}$, et nul doute que cette fiction d'aventures médiévales n'est pas là simplement pour amuser les enfants avec un nouvel avatar de la figure de Robin des Bois : elle a aussi pour vocation, dans le cadre des missions de la télévision de service public, de participer à l'édification de ce « récit commun sur l'histoire nationale ${ }^{45} »$ dont parle Françoise Lantheaume. Dans la «note de projection » de 1965 citée précédemment, on pointait la médiocrité de certaines intrigues et on ajoutait que « certaines indications de caractère éducatif (mœurs, traditions, etc.) [...] nourriraient utilement le sujet ${ }^{46} »$. À propos des aventures du chevalier Bayard ${ }^{47}$, autre héros télévisuel destiné à la jeunesse et soumis aux critiques du «Comité de lecture des émissions pour la jeunesse ${ }^{48} »$, les experts regrettaient que l'on nage « en pleine convention ${ }^{49}$ » et que l'on soit trop éloigné du personnage historique. Thierry la Fronde, du fait des moyens limités qui sont les siens, peut paraître à première vue très éloigné de telles préoccupations. La volonté de s'appuyer sur une certaine vraisemblance historique n'est pourtant pas absente du projet. Jean-Claude Deret affirme avoir beaucoup travaillé à partir de chroniques d'époque, notamment celles de Froissart, et de la presse de vulgarisation historique qu'il lisait dans sa jeunesse.

Comme en témoigne le très long texte - il dure près de deux minutes ! - de contextualisation historique qui introduit la série en ouverture du premier épisode, le feuilleton se veut documenté et crédible, avec des personnages et des épisodes historiquement attestés. Nous sommes en 1359 et au début de cette guerre « qui devait durer plus de cent ans ${ }^{50}$ :

1356 : Jean II a succédé à son père sur le trône de France [...]. Il est battu à Poitiers et fait prisonnier par Édouard, prince de Galles, dit le Prince Noir. Le Dauphin Charles devient Régent du Royaume, lutte contre l'envahisseur et contre les troubles fomentés par son cousin, le roi de Navarre, Charles le Mauvais.

44. $C f$. M. CRIVELlO-BOCCA, «L'écriture de l'histoire à la télévision. La mobilisation des consciences : La caméra explore le temps (1956-1966) », dans M. F. LÉvY éd., La Télévision dans la République. Les années 50, Paris, 1999, p. 89-105.

45. F. LANTHEAUME, «Introduction », dans EAD. et J. LÉTOURNEAU éd., Le Récit du commun. L'histoire nationale racontée par les élèves, Lyon, 2016, p. 5-12 (p. 7).

46. Pierrefitte-sur-Seine, AN, Fonds ORTF, 20130366/199.

47. Diffusion en 1964. Voir supra, n.9.

48. Ce comité n'avait à superviser que les productions « maison » de la RTF. En tant que co-production, Thierry la Fronde n'eut pas à se soumettre à ses avis.

49. Pierrefitte-sur-Seine, AN, Fonds ORTF, 20130366/25.

50. Extrait du texte déroulant de contextualisation initial avant le début du premier épisode. 
1359 : La Sologne n'a pas été épargnée. Sous la conduite du connétable Chandos, les troupes du Prince Noir pillent, ravagent, occupent cette contrée déjà pauvre. Et dans la France meurtrie et que ne peut sauver une trêve qu'aucun des partis n'entend respecter, de jeunes chevaliers tentent ce qui paraît impossible : secouer le joug de la domination anglaise [...].

Un certain nombre des personnages historiques vont sortir de ce texte déroulant pour croiser la route de Thierry la Fronde : celui-ci va rencontrer en de multiples occasions le roi Édouard d'Angleterre, son fils le Prince Noir ${ }^{51}$, le Dauphin de France et son cousin et éternel rival, le roi de Navarre Charles le Mauvais. Du Guesclin est un personnage récurrent qui s'entretient à plusieurs reprises des affaires du royaume avec Thierry. Ce sont là des «personnages référentiels » que tout le monde (re)connaît parce qu'ils ont été « $\operatorname{appris}^{52}$ » (ou sont censés l'avoir été à l'école) et qui authentifient le récit en lui donnant un ancrage historique incontestable. Mais à côté de ces grandes figures, il y a, assurément moins connus du grand public mais tout aussi historiquement attestés, des personnages secondaires comme les connétables anglais Chandos puis Captal de Buch $^{53}$ (à partir de la deuxième saison). Parmi les personnages qui témoignent d'une connaissance historiquement assez pointue de la part des scénaristes, on trouve Arnaud de Servol ${ }^{54}$, dit « l'Archiprêtre », qui, à la tête de la « compagnie blanche », cherche à enrôler Thierry dans sa lutte contre les Anglais (E20). Arnaud de Servol fut lieutenant général du roi en Berry et Nivernais et chambellan de Charles V. Ses méthodes très controversées (massacres, pillages, rançons), qui furent celles des «grandes compagnies », déplaisent fortement à Thierry qui lui reproche la rançon de 40000 écus qu'il a exigée du pape pour épargner Avignon et la Provence ${ }^{55}$. Enfin, parmi ces grands ou petits personnages historiques, Thierry va côtoyer... celui qui en fut le premier « chroniqueur », Froissart lui-même, présenté dans l'épisode 22 intitulé «La chronique oubliée » comme le secrétaire et chroniqueur de la reine d'Angleterre qui veut à tout prix rencontrer le célèbre Thierry la Fronde...

51. Son portrait dans P. Contamine, La Guerre de Cent Ans, Paris, 2016, p. 36-37.

52. P. HAMON, «Pour un statut sémiologique du personnage», dans R. BARTHES, W. KAYSER, W. C. Booth et ID., Poétique du récit, Paris, 1977, p. 115-180 (p. 122).

53. L'exemple du Captal de Buch - alias Jean de Grailly, noble gascon qui combat pour les Anglais, seigneurs de l'Aquitaine (de capdàn en gascon qui signifie « seigneur principal » et Buch qui désigne le bassin d'Arcachon) - illustre bien la complexité d'un conflit qui ne peut évidemment se résumer à un affrontement national entre Anglais et Français. Mais la série n'entre évidemment pas dans ce genre de subtilités...

54. C'est la graphie qu'adopte le générique pour le nom de ce personnage historique, plus communément orthographié Arnaud de Cervole.

55. Sur les « Grandes Compagnies » et Arnaud de Cervole, $c f$. P. ConTAMine, La Guerre de Cent Ans..., p. 52. 
La série n'hésite d'ailleurs pas à faire jouer un rôle décisif à Thierry dans le déroulé de certains faits historiques (plus ou moins) avérés. C'est ainsi que, dans l'épisode 13, il sauve le Dauphin d'une tentative d'assassinat de la part de son cousin Charles le Mauvais. Dans le numéro de Télérama qui présente la série, Jean-Claude Deret, évoquant Froissart et d'autres chroniqueurs pour certifier l'authenticité du contexte historique, affirme que l'une de ces chroniques rapporte que « le roi de Navarre essaya d'empoisonner son cousin le dauphin de France. Celui-ci ne fut sauvé que parce que le repas fut interrompu ${ }^{56} \gg$. Interrompu par Thierry, dans la série, bien entendu... Plus rocambolesque encore : dans l'épisode 18, en route pour Pontorson où ils doivent remettre un trésor de guerre à Du Guesclin, Thierry et ses compagnons, déguisés en comédiens, se retrouvent à devoir jouer pour le roi d'Angleterre : pendant un orage, et croyant à une injonction divine, le roi Édouard entend par le conduit de cheminée une conversation entre deux des compagnons - conversation dans laquelle il est question de « prendre une décision » et de « paix »- qui le décide à renoncer au trône de France et à écouter ses conseillers qui lui demandaient de mettre fin à la guerre... Dans l'épisode 27, Thierry représente le Dauphin aux pourparlers de paix de Brétigny, avant de rallumer le conflit à l'issue de l'épisode suivant.

Quant à la reconstitution d'époque - décors, costumes, mobiliers et accessoires de toutes sortes-, elle s'appuie, aux dires du créateur de la série, sur la lecture attentive du Dictionnaire raisonné du mobilier français : «On ne peut pas faire de film médiéval sans Viollet-le-Duc ${ }^{57}$. » Jean-Claude Deret affirme avoir trouvé l'idée et le modèle de la fronde de Thierry dans cet ouvrage. Il assume totalement le mélange des époques médiévales. C'est ainsi que, concernant le collant du héros qui a souvent provoqué des commentaires ironiques, il s'explique : «Il a des collants. C'est possible. C'est possible d'après Viollet-le-Duc d'avoir des collants. Il a des petites bottes courtes, c'est encore possible, bien que ce soit plus fin du XIV et début du XV $\mathrm{XV}^{\mathrm{e} 58}$. » S'il arrivait que les auteurs aient parfois un souci quasi maniaque de la vraisemblance historique comme dans le synopsis de l'épisode 46, où ils justifient scrupuleusement l'utilisation des «jouets dangereux» du titre - comprenons, des armes innovantes pour l'époque - par des références qui se veulent précises ${ }^{59}$, la vision de gouttières très modernes mais mal occultées dans les scènes de rue (au

56. Télérama, $\mathrm{n}^{\circ} 720,3$ novembre 1963, p. 17.

57. « Entretien JCD/SCD».

58. Ibid.

59. Par exemple, «Miroirs concaves pour incendier à distance les objectifs repérés à la longue vue (Roger Bacon 1275)» (Pierrefitte-sur-Seine, AN, Fonds ORTF, 20130366/265). 
demeurant relativement rares) vient cependant ruiner tout effort de réelle « homochronie ${ }^{60} »$ dans la reconstitution...

On le voit, si le contexte historique, avec ses acteurs principaux et quelques-uns de ses événements majeurs, est respecté, la «chronique » elle-même prend évidemment de grandes libertés, qui sont celles, légitimes, de la fiction historique quand elle ne se prend pas tout à fait au sérieux. Les scènes de violence bénéficient d'un traitement très édulcoré et les combats sont parfois traités de manière humoristique, avec par exemple des soldats anglais qui passent par les portes et fenêtres (E2)... comme les Romains dans les bandes dessinées d'Astérix, un autre « irréductible Gaulois » très en vogue dans la même décennie ! Nous ne sommes pas là dans le docudrama, à forte valeur documentarisante, tel que la télévision en produira ultérieurement ${ }^{61}$, mais dans ce qui est ouvertement présenté comme une série d'aventures en costumes et un divertissement à coloration historique.

\section{Thierry « maquisard »}

L'apport proprement historique de la série, sans être totalement négligeable, est donc limité mais s'inscrit dans une logique qui est celle d'une télévision nationale de service public.

La fonction éducative de cette télévision est a fortiori plus évidente encore quand il s'agit du jeune public, qu'il convient d'initier aux grandes heures de notre Histoire, sans heurter sa sensibilité. Pour les écoliers des années 1960, Du Guesclin «qui servait bien son seigneur le roi Charles et la France sa patrie ${ }^{62} »$, les bourgeois de Calais et Jeanne d'Arc sont des héros familiers exaltés par les manuels de l'école républicaine, qui transforment une multitude de conflits complexes et étirés dans le temps en une guerre de Cent Ans en forme d'épopée nationale de résistance à l'ennemi héréditaire... Or la guerre de Cent Ans, depuis Michelet et sous la houlette des historiens de la Troisième République, est perçue comme « un moment décisif d'affirmation de la conscience nationale » et, dans la France de l'après Seconde Guerre mondiale, cette période suscite un intérêt renouvelé auprès du public «en relation évidente avec les épreuves de l'histoire récente ${ }^{63} »$.

Quand le premier épisode de Thierry la Fronde est diffusé, moins de vingt ans se sont écoulés depuis la fin du conflit et les auteurs de la série

60. R. JOMAND-BAUdRY, «Présentation», dans ID. et M. NUEL éd., Images cinématographiques du siècle des Lumières, Paris, 2012, p. 7-23 (p. 12).

61. Par exemple, 1788 de M. FAILEVIC en 1978.

62. E. LAVISSE, Histoire de France, Cours élémentaire [1913], édition augmentée, Paris, 2014, p. 67.

63. F. AmY DE LA BretÈQue, Le Moyen Âge au cinéma. Panorama historique et artistique, Paris, 2015, p. 162. C'est nous qui soulignons. 
ne cachent pas leur intention de faire de Thierry de Janville un héros de la Résistance, qui prend le maquis contre l'occupant - anglais autrefois, allemand plus récemment; comme le dit Sabine Chalvon-Demersay, « la façon dont Thierry la Fronde prend le maquis en Sologne est tout à fait transparente pour des contemporains ${ }^{64} \gg$. Jean-Claude Deret le dit luimême sans ambages à plusieurs reprises : Thierry est un « maquisard de la guerre de Cent Ans ${ }^{65}$ », qui « défend son pays et prend le maquis, comme il n'y a pas si longtemps les jeunes gens le faisaient. Thierry fait donc de la résistance ${ }^{66}$. » Une guerre, une « résistance », peut donc en cacher une autre et, selon un procédé qui lui est très familier, la fiction historique s'empare du passé pour mieux nous parler du présent, élargissant alors sensiblement le public-cible initial des enfants et adolescents. En ces temps où la «petite lucarne » pénètre peu à peu dans les foyers français, on regarde la télévision en famille et, bientôt, « les adultes regardent», ce qui oblige les auteurs à rendre le propos un peu plus «musclé ${ }^{67}$ » (notamment en termes de représentation de la violence légitime du héros) et transforme Thierry en héros national, tous âges confondus.

Dans le premier épisode de la série, le jeune Thierry de Janville envisage de traverser la Manche pour porter secours à son roi prisonnier des Anglais après la bataille de Poitiers et, lorsque ceux qui vont devenir ses compagnons évoquent la situation à laquelle ils se trouvent confrontés, l'un d'entre eux s'interroge et interpelle son ami en ces termes : «Qui est ton roi ? Est-ce que c'est Jean, qui est à Londres, prisonnier ? Ou Édouard III, qui est en France, libre ?» Bien entendu, le dialogue est d'abord historiquement interprétable en relation avec la captivité de Jean le Bon et la présence des troupes d'Édouard sur une partie du territoire français, mais comment ne pas voir l'allusion à la question de légitimité du pouvoir qui s'est posée à la France de $1940^{68}$, entre l'homme du 18 juin exilé volontaire à Londres et celui qui prétendait assurer, ici et maintenant, la pérennité de l'État français ? Par ailleurs, est-ce un hasard si ce même premier épisode de la série fait allusion à un crime de guerre des troupes du connétable Chandos, qui auraient enfermé dans une église des femmes, des enfants et des vieillards avant de mettre le feu à l'édifice... ? Certes, la guerre de Cent Ans eut son lot infini de pillages, massacres et autres horreurs en tout genre, mais le téléspectateur des années 1960 pouvait-il ne pas penser ici à l'abominable tragédie d'Oradour-sur-Glane, en 1944, dont

64. S. CHALVON-DEMERSAY, «L'homme qui voulait être acteur... », p. 83.

65. Télé 7 jours, $\mathrm{n}^{\circ} 189,2$ novembre 1963, p. 26.

66. Télérama, $\mathrm{n}^{\circ} 720,3$ novembre 1963, p. 17.

67. «Entretien JCD/SCD».

68. Pas immédiatement puisqu'on sait que très peu de Français eurent la possibilité d'écouter l'appel du 18 juin, auquel seule la presse régionale de la zone libre donna quelque écho. 
un certain nombre de responsables furent jugés au début des années 1950 et dont le site fut érigé dès l'après-guerre en « lieu de mémoire » ? La France gaulliste du début des années 1960 célèbre la geste de ceux qui résistèrent à l'Occupation allemande et la télévision consacre en Thierry de Janville un héros national qui, avec sa fronde, combat les géants qui brûlent les femmes et les enfants dans les églises... À la fin de ce premier épisode, Thierry et ses premiers compagnons se réfugient dans la forêt et deviennent des « Hors-laloi » (titre de ce premier épisode), confrontés rapidement à des problèmes de ravitaillement (E3), comme vingt ans auparavant les réfractaires au STO qui allaient devenir les premiers maquisards...

De la barbarie anglaise, qui n'est pas sans évoquer celle des nazis, il sera évidemment beaucoup question dans la suite de la série, même si c'est toujours de façon allusive ou très édulcorée en matière de représentation, public jeunesse oblige. Dans l'épisode 28, les Anglais veulent raser le village en matière de représailles après l'assassinat de deux soldats anglais et s'y emploient au mieux en pillant les maisons et en massacrant une partie de la population (avant l'intervention opportune de Thierry, bien entendu). De la torture, il est également souvent question, même si elle n'est pas l'apanage des seuls Anglais. Dans l'épisode 13, les partisans du Dauphin administrent la question à un comploteur au service de Charles le Mauvais, mais dans l'épisode 14, «Les reliques », ce sont bien des soldats anglais qui fouettent au sang un homme qui a essayé de percer le secret des reliques que transporte le mystérieux convoi qui traverse la forêt solognote. L'homme supplicié a le dos zébré de coups et crie de douleur ; on comprend qu'il est censé mourir des coups qu'on lui administre car les soldats, à l'invitation de leur chef, l'abandonnent bientôt à son triste sort. Dans les épisodes 33 et 35, Thierry est victime à son tour de tortures, dont la mise en scène provoque d'ailleurs des réactions indignées de certains téléspectateurs qui pensent que ces scènes « risquent d'imprimer dans l'esprit [des] enfants la vision atroce d'une humanité bestiale et cruelle ${ }^{69} »$. Cette violence que l'on ne saurait voir fut celle de la «question » médiévale, mais aussi celle des prisons de la Gestapo ${ }^{70} \ldots$

À cette violence et à cette barbarie qui sont essentiellement celles de l'occupant, comment faut-il résister? Thierry est un héros sans peur et surtout sans reproche : c'est un héros aux mains propres et (presque) nues qui se bat avec une extrême loyauté et dans le respect de l'ennemi, qui d'ailleurs le lui rend bien. Dans l'épisode 3, Chandos se montre très méprisant envers messire Florent, l'ennemi juré de Thierry et collaborateur

69. Lettre d'une téléspectatrice, soutenue par la Commission diocésaine de Versailles (Pierrefitte-sur-Seine, AN, Fonds ORTF, 20130366/199).

70. Il s'agit assurément là de deux usages très différents de la violence mais l'une renvoie assurément à l'autre dans l'esprit du « grand public » auquel s'adressait la télévision. 
des occupants, avant de louer la noblesse d'un « ennemi qu'on pourrait se prendre à aimer »... Sa violence - nécessaire, donc légitime - ne peut être qu'une violence de rétorsion, marquée du sceau de la légitime défense. L'esprit de vengeance lui est inconnu et, quand il châtie le méchant, il le fait sans haine et, bien entendu, sans plaisir.

D'ailleurs, cette violence ne doit être convoquée que lorsque tous les autres moyens ont été épuisés. Dans le premier épisode de la deuxième saison, «Les reliques » (E14), diffusé début février 1964, Thierry et ses compagnons veulent récupérer des reliques dérobées par les Anglais à la couronne de France. Pour ce faire, Thierry imagine un stratagème très complexe, qui permet de récupérer le trésor royal sans coup férir, ou presque. Or, cette ruse demande beaucoup de travail et d'ingéniosité : il faut notamment confectionner une châsse totalement identique à celle dans laquelle se trouvent les reliques afin de procéder à une substitution, afin que les Anglais n'y voient que du feu... L'affaire est si compliquée à mettre en œuvre que l'un des hors-la-loi fait remarquer à son chef qu'il serait peut-être plus facile d'attaquer, tout simplement, le convoi... Thierry s'y refuse, et on le voit à la fin de l'épisode prendre des risques pour restituer aux Anglais ce qu'ils croient être la « vraie » châsse contenant les reliques... alors que celles-ci sont déjà dans les mains de ses compagnons. Du panache avant toute chose... Jamais le héros ne se compromet dans « les formes équivoques de la violence » que dénoncent Télérama et ses lecteurs lors d'une grande campagne en 1965. C'est pourquoi il ne saurait cautionner les méthodes plus que douteuses de ceux qui prétendent combattre l'occupation anglaise par le massacre, le pillage et la rançon, comme ce capitaine de « grande compagnie », Arnaud de Servol, qu'il croise dans l'épisode 20, ou encore ces «Tuchins » (E33), bandits de grand chemin aux méthodes également brutales et que combat Thierry avant de les rallier à la bonne cause du Dauphin... Thierry ne peut avoir les mains sales et la fin ne saurait justifier les moyens, la situation fût-elle des plus périlleuses. En 1961, le téléfilm d'inspiration sartrienne de Maurice Cazeneuve, L'Exécution, qui justifia par ailleurs aux yeux de la RTF la mise en place du carré blanc, provoqua le malaise car, non content de montrer aux téléspectateurs médusés le « dos » d'une femme nue, il mettait en scène un groupe de résistants qui jugeait sommairement et exécutait laborieusement, en une séquence interminable, un collaborateur susceptible de mettre en danger leur réseau ${ }^{71}$. Il y avait là une atteinte intolérable aux yeux de certains au mythe de la Résistance et à son héritage que gaullistes et communistes se disputaient alors âprement : rien ne devait porter atteinte au « résistancialisme ${ }^{72}$ » ambiant

71. B. PAPIN, L'Invention du carré blanc. Images convenables, images inconvenantes sur le petit écran des années soixante, Paris, 2006.

72. H. Rousso, Le Syndrome de Vichy... 
qui véhiculait l'image d'une France unanimement résistante et d'une armée des ombres aux méthodes toujours irréprochables. Il était donc tout à fait inenvisageable que le Robin des Bois de Sologne ait recours à des actes que l'on eût pu qualifier de « terroristes », à l'instar de l'occupant allemand, au temps notamment des assassinats ciblés de soldats allemands.

La «collaboration » et «l'épuration» qui s'ensuivit ne sont pas absentes non plus. Dans l'épisode 28, alors qu'un accord de trêve vient d'être signé à Brétigny, Thierry la Fronde redevient Thierry de Janville et ses compagnons, démobilisés, rentrent au village ; « la paix commence » avec son cortège de controverses sur le rôle de chacun pendant les temps troublés de l'occupation... Un mauvais bougre, le sorcier Germain, après avoir accusé Thierry et ses hors-la-loi de s'être « cachés » pendant la guerre, joue les résistants de la dernière heure et fait pendre par la foule deux déserteurs anglais... Il accuse le tavernier, ami de Thierry et sympathisant de la résistance armée contre les occupants, d'avoir collaboré avec l'ennemi - « Tu les as toujours bien reçus chez toi, les Anglais, hein ?» - avant de chercher à le faire pendre également. Pendant ce temps, les villageois ont également saccagé le château de Janville, où résidaient messire Florent et le commandement anglais... Comme vingt ans auparavant dans la France de la Libération, on solde donc ici les comptes de l'occupation.

On sait, au moins depuis les travaux de Pierre Sorlin et Marc Ferro, que la fiction historique, fût-elle la plus scrupuleuse, ne peut guère échapper aux «lapsus ${ }^{73}$ » qui trahissent l'époque qui l'a vue naître. Marc Ferro va jusqu'à affirmer qu'il n'a jamais «considéré les films historiques comme des révélateurs sur l'Histoire. Sauf sur l'histoire de l'époque où ils sont faits ${ }^{74}$.»Un certain nombre de cinéastes, refusant la « reconstitution » historique, revendiquent même le droit de transformer leurs récits historiques en manifestes politiques contemporains : le « médecin des Lumières » de René Allio (Un médecin des Lumières, 1988) est de son propre aveu plus un moderne « intellectuel de gauche » qu'un médecin philosophe du temps des Lumières, et ses «camisards » (Les Camisards, 1972) ressemblent farouchement à des guérilleros des années 1970 75 . Et lorsque Andrzej Wajda, dans les années 1980, met en scène la lutte à mort entre Danton et Robespierre (Danton, 1982), les commentateurs ne s'y trompent pas : si son

73. M. Ferro, Cinéma et Histoire, Paris, 1993, p. 74.

74. M. FERro, Mes histoires parallèles. Entretiens avec Isabelle Veyrat-Masson, Paris, 2011, p. 266.

75. B. PAPIN, " "Librement inspiré par les travaux de..." : la "relation dialectique" de l'artiste et du savant dans l'écriture scénaristique des fictions historiques de René Allio », dans M. Scheinfeigel et M. TsikounAs éd., René Allio. Écrits d'écran, Paris, 2018, p. 93108. 
film respecte dans ses grandes lignes les événements révolutionnaires, il nous parle «surtout de la Pologne de 1982 et du communisme ${ }^{76}$ ».

La fiction historique nous en dit toujours autant, sinon plus, sur la période qui la produit que sur celle qu'elle prétend mettre en scène. C'est l'Occupation allemande de 1940 qui est lisible derrière l'occupation anglaise de la Sologne de 1359. Nul doute que les téléspectateurs adultes des années 1960 ont su percevoir, derrière les bondissantes (pas toujours) mais (souvent) répétitives aventures du frondeur, les stigmates d'une histoire récente et douloureuse. Aux yeux du public adulte qui avait rejoint le cortège des jeunes fidèles de la série de Jean-Claude Deret, le château de Janville occupé prenait des airs de Kommandantur, la forêt solognote évoquait les maquis du Limousin ou du plateau des Glières, les villages martyrisés étaient autant d'Oradour-sur-Glane... Et, dans une France gaulliste qui a du mal à mettre en œuvre les utopies du Conseil National de la Résistance, il faut vanter la lutte d'un héros aux mains propres qui se bat au nom de tous et incarne de ce fait l'unité nationale - une unité nationale mise à mal par l'agonie de la Quatrième République et l'enfantement dans la douleur de la Cinquième.

Pour incarner cet esprit de résistance, il convenait de mettre en scène « un gentil », « un pur ${ }^{77}$ », qui respecte ce que le rapport Kriegel de 2002 sur « la violence à la télévision » appelle la « logique du champ de bataille ${ }^{78}$ », où s'exerce une violence régulée, motivée et donc exemplaire. La musique du générique de Thierry la Fronde est restée dans toutes les mémoires. Les paroles de La Marche des compagnons, écrites par Jean-Claude Deret - qui furent chantées par John William ${ }^{79}$, un produit « dérivé » parmi d'autres ${ }^{80}-$, en sont moins connues mais comment ne pas voir que cet appel à la mobilisation des «soldats de l'ombre », notamment par son interpellation initiale, fait écho à un plus célèbre encore «Chant des partisans »?
Ami le temps n'est plus aux chansons.
Il faut partir, laisser ta maison.
Et tout quitter, jusqu'à son nom.
Compagnon! Compagnon!

76. Voir l'analyse du film par G. CHAUBERT sur le site de l'association Thucydide : [http://www.thucydide.com/realisations/voir/analyses/danton.htm].

77. « Entretien JCD/SCD ».

78. B. KRIEGEL éd., La Violence à la télévision. Rapport de la mission d'évaluation, d'analyse et de propositions relatives aux représentations violentes à la télévision, Paris, 2003, p. 52.

79. John William, qui connut une grande célébrité comme chanteur dans les années 1960, avait été arrêté en 1942 pour fait de résistance, torturé puis déporté : [https://www.ouestfrance.fr/europe/france/john-william-le-chanteur-de-thierry-la-fronde-est-mort-627066].

80. [https://www.youtube.com/watch?v=wsHdEvpgrko] 
Sur le chemin que nous avons pris.

Soldats de l'ombre, enfants de la nuit.

Que l'ennemi craigne ton nom.

Compagnon! Compagnon!

Tu auras faim et tu auras froid.

Tu marcheras et n'oublieras pas.

Il faut se battre pour la liberté.

Gagner la guerre et gagner la paix.

Le temps est court, le chemin si long.

Compagnon! Compagnon ! [...]

Bernard Papin - CIM, Université de la Sorbonne Nouvelle

Thierry la Fronde, figure de la Résistance. Quand une Occupation en cache une autre...

Dans la France du début des années 1960, une série médiévale destinée au jeune public triomphe à la télévision: Thierry la Fronde (1963-1966) conte les aventures d'un jeune noble, Thierry de Janville, qui s'oppose à l'occupation anglaise de la Sologne pendant la guerre de Cent Ans. Très vite cependant, ce Robin des Bois solognot fédère autour du petit écran alors très familial un public beaucoup plus large que celui initialement visé et cette série, historiquement approximative et scénaristiquement plutôt médiocre, devient un immense succès. Comment expliquer que cette fantaisie historique pour adolescents ait pu susciter un tel engouement? La réponse est sans doute à chercher dans la transformation du frondeur médiéval en moderne " maquisard " et c'est l'Occupation allemande de 1940 qui est lisible derrière l'occupation anglaise de la Sologne de 1359. Une vingtaine d'années après la fin de la Seconde Guerre mondiale, les téléspectateurs adultes ne s'y sont pas trompés et ont su discerner, dans les lointaines aventures historiques proposées à leurs enfants, les stigmates d'une histoire plus récente et tout aussi douloureuse.

guerre de Cent Ans, médiévalisme, Occupation allemande, Résistance, séries télévisées, télévision, Thierry la Fronde. 


\section{Thierry la Fronde, Figure of the Resistance. Occupied France Revisited}

In France in the early sixties, a medieval TV series aimed at young audiences triumphed on television: Thierry la Fronde (1963-1966) told the adventures of a young nobleman, Thierry de Janville, who opposed the English occupation of Sologne during the Hundred Years' War. Very quickly, however, this local Robin Hood gathered a much wider audience than originally targeted at a time when watching TV was essentially a family pastime. This series, which was loosely historical and relied on rather mediocre scenarios, became a huge success. Why did this historical fiction TV series for teenagers attract such tremendous interest? Probably because the medieval stone-slinging rebel became a metaphor for a modern "maquisard" and a symbol of the French Resistance in the Second World War, while the portrayal of the English occupation of Sologne in 1359 was very much reminiscent of the German Occupation of 1940 . With the series being broadcast about twenty years after the end of the war, adult viewers picked up on this symbolism, recognizing behind the distant historical adventures offered to their children the stigmata of a more recent and equally painful story.

German Occupation of France, Hundred Years' War, Resistance, television, Thierry la Fronde, TV series. 
